\title{
Detection of a slow-flow component in contrast-enhanced ultrasound of the synovia for the differential diagnosis of arthritis
}

\author{
Gaia Rizzo, Matteo Tonietto, Marco Castellaro, Bernd Raffeiner, Alessandro Coran, Ugo Fiocco, \\ Roberto Stramare and Enrico Grisan
}

\begin{abstract}
Contrast Enhanced Ultrasound (CEUS) is a sensitive imaging technique to assess tissue vascularity, that can be useful in the quantification of different perfusion patterns. This can particularly important in the early detection and differentiation of different types of arthritis. A Gamma-variate can accurately quantify synovial perfusion and it is flexible enough to describe many heterogeneous patterns. However, in some cases the heterogeneity of the kinetics can be such that even the Gamma model does not properly describe the curve, especially in presence of recirculation or of an additional slow-flow component.

In this work we apply to CEUS data both the Gamma-variate and the single compartment recirculation model (SCR) which takes explicitly into account an additional component of slow flow. The models are solved within a Bayesian framework.

When dividing the patients into two groups (rheumatoid arthritis and polyarticular RA-like psoriatic arthritis vs. other arthritis types), the median value of the parameter indicating the presence of slow-flow is significantly different $(0.12 \mathrm{vs} 0.09$, $p<0.002)$, whereas when performing ANOVA to check the differences among each type of arthritis, the median is no more different, but the parameter distribution is $(\mathbf{p}<0.05)$.
\end{abstract}

\section{DESCRIPTION OF PURPOSE}

Contrast-enhanced Ultrasound (CEUS) is an imaging technique that uses microbubbles as contrast agent to visualize and assess tissue vascularization and perfusion. In particular, CEUS has been widely used in the study of the arthritis disease to non-invasively assess synovial neo-vascularization and local perfusion variations for early detection and grading of the pathology [1-5]. CEUS data are generally quantified at the region of interest (ROI) level, i.e. analyzing the time activity curve (TAC) obtained by averaging all pixel TACs within a specific user-defined region. In a previous work, we have tackled the CEUS quantification problem at the pixel level and we have demonstrated that pixel-wise quantification allows such an effective characterization of different perfusion patterns that they can be then used to discriminate between arthritis subtypes [6]. Moreover, we showed that a physiologically motivated perfusion curve, such as the Gamma-variate model, can accurately quantify synovial perfusion and it is more flexible than a mono-exponential or logarithmic model (generally employed for CEUS kinetic description) to describe many heterogeneous patterns. However, in some cases the heterogeneity of the CEUS kinetics can be such that even a Gamma-variate model does not properly describe the time courses, as in presence of an apparent trapping of the microbubbles in newly formed vasculature, when characterized by high tortuosity [7,8]. This apparent trapping can also be explained by the limited temporal duration of the CEUS scan, that prevents the identification of a very slow washout from the data. This problem has already been considered in other contrast-based imaging techniques (e.g. in dynamic susceptibility contrast magnetic resonance imaging, DSC-MRI), where the original Gamma-variate was augmented with its integral to explicitly model the recirculation of the contrast agent (single compartment recirculation, SCR) $[9,10]$. However, nothing similar has ever been investigated for CEUS images quantification.

In the current work, we solve both the Gamma model and the SCR model at pixel level within a Bayesian framework using Variational Bayesian estimator, already applied to MR [11] and positron emission tomography (PET) data [12]. Prior information for the pixels were generated automatically from the image data using the estimates obtained by applying the model to a set of regional time courses solved with a nonlinear estimator in a hierarchical, user-independent, approach $[12,13]$.

G.T., M.T., M.C., and E.G. authors are with the Department of Information Engineering, University of Padova, Padova, Italy. B.R. and U.F. and R.S. authors are with the Department of Medicine, University of Padova, Padova, Italy

A.C. is with the IRCCS Veneto Institute of Oncology, Padova, Italy 


\section{METHODS}

The classical Gamma-variate model will consider negligible any irreversible component of the contrast agent, compared to the general picture of the system dynamics.

An alternative model to describe CEUS kinetics is the single recirculation component (SCR), previously proposed for DSC-MRI perfusion imaging. This model can be used to describe the irreversible component in CEUS:

$$
c_{S C R}(\boldsymbol{p}, t)=c_{\text {gamma }}(t)+\frac{a_{1}}{A U C_{g}} \int_{t_{0}}^{t} c_{\text {gamma }}(\tau) d \tau t \geq t_{0}
$$

with the parameters $\boldsymbol{p}_{\boldsymbol{S C R}}=\left[a_{0}, \alpha, t_{0}, \beta, a_{1}\right]$ where $a_{1}$ is the scaling factor (normalized by the area under the curve $A U C_{g}$ of $c_{\text {gamma }}(t)$ to range between 0 and 1 . The integral term describes the slow-flow in the synovial perfusion.

\section{Variational Bayes estimation}

In the Bayesian framework, we want to estimate the posterior distribution of the parameters $P(\boldsymbol{\Theta} \mid \boldsymbol{y}, m)$, where $\boldsymbol{\Theta}$ is the vector of the parameters of a chosen model $m$ from a set of measured data $\boldsymbol{y}$. In real applications, the numerical integrations needed for the direct computation of the posterior are usually intractable. Here we implement a Variational Bayesian (VB) approach, already proposed for medical imaging analysis [11,12], which, in brief, exploits a mean field approximation of the true posterior, i.e. $Q(\boldsymbol{\Theta} \mid \boldsymbol{m})=\sum_{i} Q_{i}\left(\boldsymbol{\Theta}_{i} \mid \boldsymbol{m}\right)$. The problem therefore shifts to the minimization of the divergence between the approximated and the true posterior, which is achieved by maximizing a quantity called Free Energy (which acts as the lower bound of the log model evidence, that can be used for model selection [16]).

A key point in Bayesian modeling is the prior definition: we implemented an automatically user independent approach to derive the priors from the data. We defined the prior distributions as a multivariate Gaussian distribution for the vector of model parameters $\boldsymbol{p}$ and an uninformative Gamma distribution for the variance scaling factor $\phi$. The priors for the model parameters were obtained from a preliminary estimation on 6 regions of interest within the synovia, identified by functional clustering (k-means partitioning method $[14,15])$ and setting the variance of the priors as $50 \%$ of the estimated mean priors value:

$$
P(\boldsymbol{p}) \sim \mathcal{N}\left(\boldsymbol{p} ; \boldsymbol{p}_{0},\left(0.5 \boldsymbol{p}_{\mathbf{0}}\right)^{2}\right)
$$

\section{DATASET}

Data from previously reported studies [6] of 115 subjects affected by arthritis were used in the current study. The cohort included 53 patients with rheumatoid arthritis and 58 patients with non-rheumatoid (non-RA). Among the non-RA patients, 21 had distal predominant (dpPSA) and 9 polyarticular RA-like psoriatic arthritis (PSA), 8 undifferentiated spondyloarthritis (uSPA), and 8 had arthritis in connective tissue disease (CTD).

All patients showed signs of inflammatory finger joint involvement: the joint with the highest disease activity was chosen for CEUS examination. All patients gave their informed consent to the examination, to the intravenous administration of the contrast agent and to the participation of the study that was approved by the local institutional ethical committee. Each subject underwent a 2-min CEUS study with a 7-MHz transducer US device (MyLab25, EsaOte) equipped with Contrast tuned Imaging (CnTI; Esaote), according to the procedure described in [17]. Gray-scale US (anatomical B-mode image) were acquired to define the boundaries of the synovial tissue (semi-automatically outlined by the radiologists as in [18]). CEUS images were motion corrected and coregistered to the anatomical image, normalized, and extracted the pixels within the synovial boundaries showing a significant enhancement [6]. We excluded sixteen subjects with no active pixels from the analysis. 


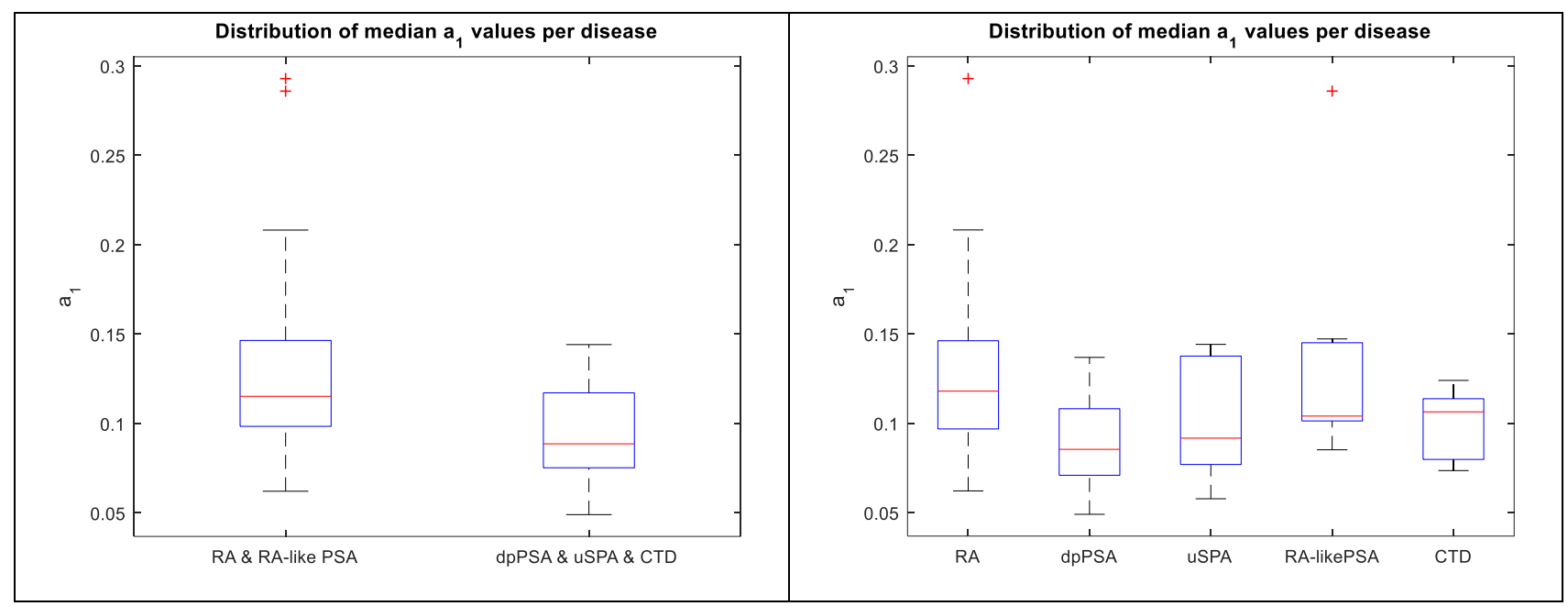

Fig. 1 Boxplots showing the empirical distribution of the estimated parameter $a_{1}$, linked to the presence and amount of a slow-flow component in the synovia. On the left panel, the RA and RA-like psoriatic arthritis are grouped together against the other, whereas on the right panel all diseases are plotted separately.

\section{RESULTS}

The estimated parameters using the SCR model allow the identification of the presence of a slow-flow component in the synovial perfusion through the quantification of the parameter $a_{1}$. The amount of this component seems related to different types of angiogenetic processes, that are characteristics of different types of arthritis diseases. When subdividing the available data into those affected by rheumatoid arthritis and polyarticular RA-like psoriatic arthritis and those affected by other types of arthritis, both t-test $(\mathrm{p}<0.002)$ and a rank-sum test $(\mathrm{p}<0.002)$ suggest that the value of $a_{1}$ is significantly different in the two populations. The distribution of its value is shown in the boxplots of Fig 1 . Then, we tried to assess by means of an analysis of variance (ANOVA) if the parameter $a_{1}$ can be significantly different in the populations identified by all types of arthritis in the data. As can be seen in Fig. 1, right panel, the median values are not significantly different among all diseases $(p=0.29)$, but the distributions of its value are $(p<0.05)$.

\section{NEW OR BREAKTHROUGH WORK TO BE PRESENTED}

A new model for the description of contrast-enhanced ultrasound data integrating a slow-flow component has been proposed and applied in the analysis of the perfusion patterns of arthritis patients. The slow-flow component seems relevant for the characterization of the different types of disease.

\section{CONCLUSIONS}

We propose a more complex model for describing the perfusion kinetics of ultrasound contrast within the synovia, accounting for a slow-flow component similar to what appears to be recirculation in DSC-MRI. The mean value of the parameter describing the amplitude of this component is able to improve the characterization of different arthritis, STATEMENT OF ORIGINALITY

This study has not been submitted, published, or presented elsewhere.

\section{REFERENCES}

[1] Klauser A, Demharter J, De Marchi A, Sureda D, Barile A, Masciocchi C, et al. Contrast enhanced gray-scale sonography in assessment of joint vascularity in rheumatoid arthritis: results from the IACUS study group. Eur Radiol. 2005;15(12):2404-10.

[2] De Zordo T, Mlekusch SP, Feuchtner GM, Mur E, Schirmer M, Klauser AS. Value of contrast-enhanced ultrasound in rheumatoid arthritis. Eur J Radiol. 2007;64(2):222-30.

[3] Grisan E, Rizzo G, Coran A, Raffeiner B, Stramare RS. Quantitative ultrasound for diagnosis and assessment of rheumatoid arthritis. SPIE Newsroom. 2015 Jul 6;

[4] Fiocco U, Stramare R, Coran A, Grisan E, Scagliori E, Caso F, et al. Vascular perfusion kinetics by contrastenhanced ultrasound are related to synovial microvascularity in the joints of psoriatic arthritis. Clin Rheumatol. 
2015;34(11):1903-12.

[5] Mouterde G, Aegerter P, Correas J-M, Breban M, D’Agostino M-A. Value of contrast-enhanced ultrasonography for the detection and quantification of enthesitis vascularization in patients with spondyloarthritis. Arthritis Care Res (Hoboken). 2014;66(1):131-8.

[6] Rizzo G, Raffeiner B, Coran A, Ciprian L, Fiocco U, Botsios C, et al. Pixel-based approach to assess contrastenhanced ultrasound kinetics parameters for differential diagnosis of rheumatoid arthritis. J Med Imaging. 2015;2(3):34503.

[7] Fearon U, Griosios K, Fraser A, Reece R, Emery P, Jones PF, et al. Angiopoietins, growth factors, and vascular morphology in early arthritis. J Rheumatol. 2003;30(2):260-8.

[8] Kennedy A, Ng CT, Biniecka M, Saber T, Taylor C, O’Sullivan J, et al. Angiogenesis and blood vessel stability in inflammatory arthritis. Arthritis Rheum. 2010;62(3):711-21

[9] Johnson G, Wetzel SG, Cha S, Babb J, Tofts PS. Measuring blood volume and vascular transfer constant from dynamic, T 2*-weighted contrast-enhanced MRI. Magn Reson Med. 2004;51(5):961-8.

[10] Patil V, Johnson G. An improved model for describing the contrast bolus in perfusion MRI. Med Phys. 2011;38(12):6380-3.

[11] Chappell M a, Groves a R, Whitcher B, Woolrich MW. Variational Bayesian Inference for a Nonlinear Forward Model. IEEE Trans Signal Process. 2009;57(1):223-36.

[12] Castellaro M, Rizzo G, Tonietto M, Veronese M, Turkheimer FE, Chappell MA, et al. A Variational Bayesian inference method for parametric imaging of PET data. Neuroimage. 2016;(under review).

[13] Rizzo G, Turkheimer FE, Keihaninejad S, Bose SK, Hammers A, Bertoldo A. Multi-Scale hierarchical generation of PET parametric maps: Application and testing on a [11C] DPN study. Neuroimage. 2012;59(3):2485-93.

[14] Rizzo G, Raffeiner B, Coran A, Stramare R, Grisan E. Data-Driven Learning to Detect Characteristic Kinetics in Ultrasound Images of Arthritis. Clinical Image-Based Procedures Translational Research in Medical Imaging. Springer International Publishing; 2014. p. 17-24.

[15] Rizzo G, Turkheimer FE, Bertoldo a. Multi-scale hierarchical approach for parametric mapping: assessment on multi-compartmental models. Neuroimage. 2013;67:344-53.

[16] Penny WD. Comparing Dynamic Causal Models using AIC, BIC and Free Energy. Neuroimage. 2012;59(1):31930

[17] Stramare R, Raffeiner B, Ciprian L, Scagliori E, Coran A, Perissinotto E, et al. Evaluation of finger joint synovial vascularity in patients with rheumatoid arthritis using contrast-enhanced ultrasound with water immersion and a stabilized probe. J Clin Ultrasound. 2012;40(3):147-54.

[18] Veronese E, Stramare R, Campion A, Raffeiner B, Beltrame V, Scagliori E, et al. Improved detection of synovial boundaries in ultrasound examination by using a cascade of active-contours. Med Eng Phys. 2013;35(2):188-94. 